\title{
i-PARKING: Sistema Inteligente para Control de Plazas de Estacionamiento en Vías Públicas de Zonas Urbanas
}

\author{
Jazmín Acosta, Juan P. Tintos, Juan A. Guerrero \\ Universidad de Colima \\ \{jazmin_acosta, jtintos1, antonio_guerrero\}@ucol.mx
}

\begin{abstract}
Uno de los problemas que afecta a las grandes zonas urbanas en relación al transporte es el estacionamiento de vehículos. En las grandes ciudades es extremadamente difícil encontrar espacios vacantes para poder estacionar los vehículos, especialmente en horas pico. Esto generalmente causa un desgaste de tiempo, una frustración para los conductores así como un problema de congestionamiento. El surgimiento en los últimos años de tecnologías emergentes como por ejemplo las redes de sensores pueden colaborar a solventar de mejor forma el problema de gestión de espacios de estacionamiento habilitando mecanismos de monitoreo eficiente $y$ proporcionando alta flexibilidad de control para este proceso, contribuyendo de esta forma al concepto denominado ciudades inteligentes (smart cities). En este trabajo se presenta $i-P A R K I N G$ un sistema inteligente basado en tecnologías emergentes para el control de plazas de estacionamiento controladas por parquímetros en vías públicas en zonas urbanas.
\end{abstract}

Keywords: estacionamiento, comunicaciones inalámbricas, VANETs, WSN.

\section{Introducción}

La sociedad moderna enfrenta serios problemas con los sistemas de transporte. El reporte publicado por el Fondo de Población de las Naciones Unidas (UNFPA) mostró que, por primera vez en la historia, más de la mitad de la población mundial (alrededor de 3.5 billones de personas) vive en zonas urbanas [1]. Dicho informe menciona que de acuerdo a esta tendencia se espera que dentro de 35 años, dos terceras partes de la población vivirán en zonas urbanas. Como consecuencia, en las últimas décadas las ciudades han sufrido un incremento desmedido del parque vehicular. De acuerdo al Departamento de Transporte de los Estados Unidos el número de vehículos dentro de ese país se incrementó de 234 millones en 2002 a 253 millones en 2011 [2]. El incremento descontrolado en urbanización hace necesario mecanismos eficientes y sustentables ambientalmente de gestión de varios procesos y operaciones urbanas. Uno de los problemas que afecta a las grandes zonas urbanas en relación al transporte es el estacionamiento de vehículos. En las grandes ciudades es extremadamente difícil encontrar espacios vacantes para poder estacionar los 
vehículos, especialmente en horas pico. Esto generalmente causa un desgaste de tiempo y una frustración para los conductores.

Algunas medidas que se han implementado en las grandes ciudades son la instalación de parquímetros en las avenidas y calles y los conductores tienen que pagar por estacionarse en las zonas públicas. Sin embargo, los conductores siguen padeciendo de pérdida de tiempo en el proceso de localización de un lugar para estacionar el vehículo. En los últimos años han surgido una serie de tecnologías emergentes que pueden colaborar a solventar de mejor forma el problema de gestión de espacios de estacionamiento habilitando mecanismos de monitoreo eficiente y proporcionando alta flexibilidad de control para este proceso, lo que contribuirá de esta forma al concepto denominado ciudades inteligentes (smart cities). En el caso de la gestión de estacionamiento el reto es manejar eficientemente los espacios disponibles y reducir el volumen de vehículos cruzando por las calles en busca de lugares de estacionamiento para aligerar no solamente el congestionamiento vial sino también los problemas relacionados con el impacto ambiental. Con base en lo anterior es como surge $i$-PARKING un sistema inteligente para la administración de espacios de estacionamiento en vías públicas de zonas urbanas.

El resto del artículo está organizado de la siguiente forma. La sección 2 presenta una serie de trabajos relacionados con el problema abordado en este artículo. iPARKING se describe en forma detallada en la sección 3. Finalmente se da cierre al artículo con las conclusiones del trabajo.

\section{Trabajos Relacionados}

En los últimos años se han redoblado esfuerzos para tratar de resolver el problema de gestión de plazas de estacionamiento. En específico, en la literatura se han publicado varios trabajos relacionados con el control de estacionamientos basados en el uso de tecnologías de comunicaciones.

En [3] los autores proponen SPARK, un sistema que hace uso de comunicación vehicular y la infraestructura de control de estacionamiento para proveer y administrar el estacionamiento completo. El principal problema es que este sistema solamente es aplicado en estacionamientos públicos y no se implementa en avenidas y calles. En [4] los autores un sistema para resolver de la mejor manera posible el problema con la sobrepoblación de autos en estacionamientos o incluso en la vía pública. Para este fin se propone un sistema inteligente de detección de autos y espacios para estacionarse basado en tecnología de sensores debido a que tiene un gran potencial de proporcionar una solución fácil y económica a esta aplicación. Sin embargo, la principal debilidad de este trabajo es que se queda corto en la explicación del mecanismo propuesto.

Otros trabajos presentan sistemas de estacionamiento inteligente que utilizan procesos estocásticos para modelar el proceso de entrada-salida de estacionamientos [5-6]. Mediante este análisis se pueden estudiar los servicios de estacionamiento óptimos y encontrar los mejores servicios. La información de los servicios se distribuye a los vehículos que pasan por el sitio de estacionamiento a través de redes inalámbricas. 
En [7] los autores proponen una solución denominada PhonePark que detecta la disponibilidad de estacionamiento en la vía pública haciendo uso de los dispositivos móviles que llevan los conductores. La solución no se basa en cualquier infraestructura dedicada o sensores externos, y por lo tanto es mucho más económico que las soluciones existentes. Finalmente en [8] presentan una propuesta para la gestión de plazas de estacionamiento, que se basa en la detección rápida y oportuna de plazas libres para estacionarse. Sin embargo no queda claro como realizan la detección rápida de plazas desocupadas.

Finalmente en [8] se propone iParkings un sistema para control de plazas en estacionamientos públicos y privados y en estacionamientos en zonas públicas de ciudades. El sistema propone la colocación de una serie de sensores inalámbricos que trabajan sin conexión, ni energía eléctrica, los conductores pueden saben mediante GPS o a simple vista, los lugares de estacionamiento disponibles en esa calle o en zonas cercanas. El principal problema que tiene este trabajo es que no presenta una explicación clara de cómo los sensores inalámbricos distribuyen la información de estado de la plaza que controlan ni la forma en como se presenta a los usuarios.

Como se puede observar existen trabajos que tratan de solventar el problema de control de estacionamientos, sin embargo la principal diferencia de este sistema es que al conductor se le enviará información de espacios de estacionamiento en la vía pública reutilizando la red de parquímetros que se tienen instalados en las zonas urbanas.

\section{Descripción de i-PARKING}

i-PARKING es un sistema que se basa en el modo de comunicación V2I(Vehicle-toInfrastructure) definido en las redes VANET (Vehicular Ad-Hoc Networks). V2I hace referencia a la comunicación entre vehículo y algún tipo de infraestructura colocada en las avenidas, la cual se le denomina RSU (Roadside Unit). $i$-PARKING define dos arquitecturas: física y lógica. La arquitectura física define toda la infraestructura que se debe de implementar para el funcionamiento del sistema. La arquitectura lógica la forman todos los módulos, entidades funcionales y protocolos de comunicación que tienen como función facilitar el procesamiento y difusión de la información dentro de i-PARKING.

\subsection{Arquitectura física}

La arquitectura física de $i-P A R K I N G$ comprende todos los dispositivos que se utilizan para el intercambio de información entre los diferentes actores que participan en $i$ PARKING (Fig. 1). En este caso se considera que los parquímetros conforman una red conectada en forma cableada o inalámbrica con conexión directa a través de Internet al servidor principal de gestión de plazas de estacionamientos.

$i-P A R K I N G$ está conformado por módulos que trabajan en forma integrada. A continuación se describen cada uno de los módulos y las funciones que realiza dentro del i-PARKING. El Servidor Administrador de Estacionamiento (SAE) es responsable de controlar en forma centralizada el estado de cada nodo monitor de parking (NMP). 
El SAE es un servidor con una aplicación de gestión de las plazas de estacionamiento. El Nodo Monitor de Parking (NMP) es un sistema embebido inalámbrico con sensores que detecta el estado de ocupación de la plaza de estacionamiento. Este nodo está localizado en cada parquímetro y mediante un sistema de información inalámbrico se envía el estado de la plaza de estacionamiento a un centro de datos que está representado por el SAE. Finalmente los Nodos móviles (NM) representan los OBU (On-Board Unit) y son los que solicitan el servicio de estacionamiento directamente al SAE mediante una aplicación móvil. Dicha aplicación está fuera del alcance de este artículo.

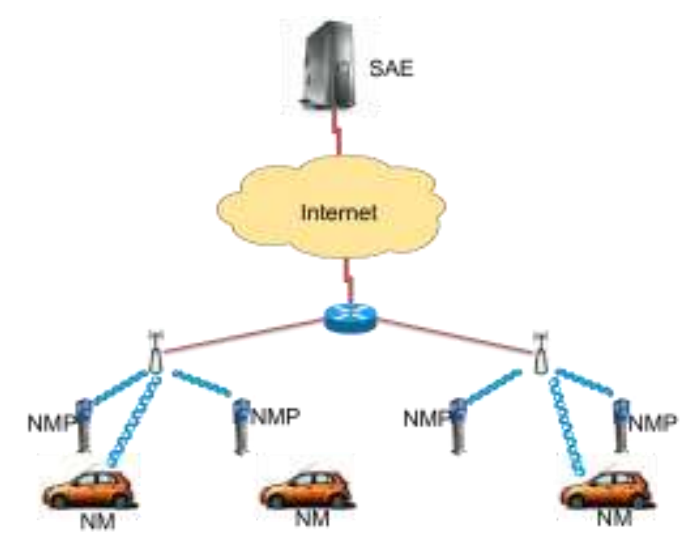

Figura 1. Representación de la arquitectura física de $i-P A R K I N G$.

\subsection{Arquitectura lógica}

La arquitectura lógica de $i-P A R K I N G$ la conforman las entidades funcionales que son implementadas en cada módulo de la arquitectura física, el protocolo de comunicación y los formatos de trama de los mensajes. A continuación se explica a detalle cada una de las partes que conforman esta arquitectura.

\subsubsection{Entidades funcionales}

$i$-PARKING está conformado por diferentes entidades que trabajan en forma integrada. A continuación se describen cada una de las entidades y las funciones que realiza dentro del i-PARKING. El Servidor Administrador de Estacionamiento (SAE) es la entidad responsable de recibir las diferentes solicitudes de las entidades NM que se encuentran implementadas en los OBU. Además es la que solicita la información de cada uno de las entidades implementadas en los módulos NMP. La entidad NMP es responsable de la información de monitoreo de la plaza asignada en el sistema de control de espacios de estacionamiento, informándole a la SAE sobre la disponibilidad e información relevante de tiempo ocupado y la información del vehículo que está en dicha plaza. Finalmente la entidad NM es la responsable de la 
comunicación directa con SAE para la solicitud de plaza de estacionamiento y de procesar la respuesta recibida de dicha solicitud.

\subsubsection{Protocolo de comunicación}

i-PARKING definen diferentes modos o procesos de comunicación para el intercambio de información entre las diferentes entidades funcionales que conforman el sistema. A continuación se describen los procesos que se incluyen dentro del sistema para una comunicación exitosa (Fig. 2).

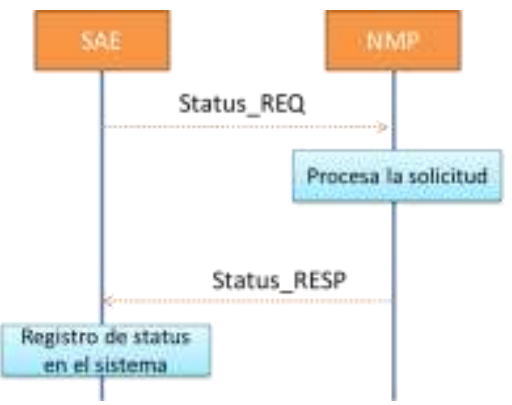

(a)

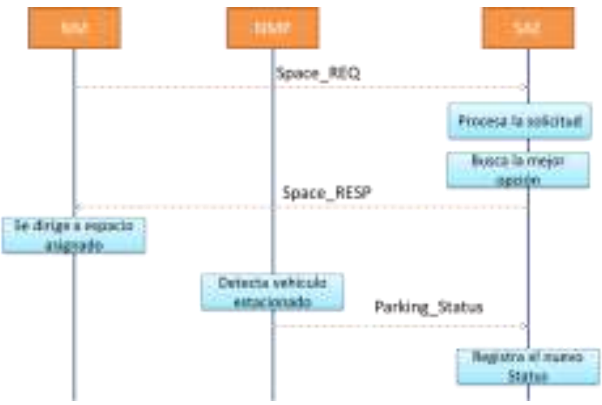

(b)

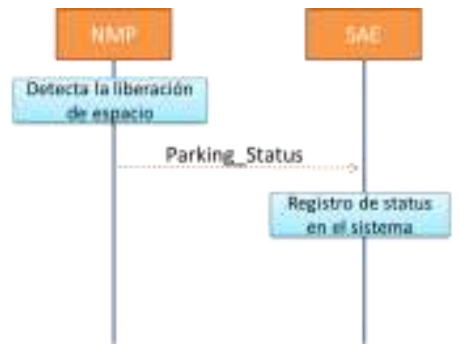

(c)

Figura 2. Representación de los procesos de la arquitectura lógica de $i-P A R K I N G$, a) proceso de inicialización del sistema, b) proceso de solicitud de plaza de estacionamiento, c) proceso de liberación de plaza.

El primer proceso definido es el de inicialización el cual se ejecuta cuando se inicializa por primera vez el sistema (Fig. 2a). En este caso el $\boldsymbol{S} \boldsymbol{A} \boldsymbol{E}$ envía un mensaje de solicitud Status_REQ mediante Broadcast dirigida a todos los NMP para que le notifiquen el estado en que se encuentra cada uno de los espacios que están gestionando. Cada uno de los NMP notifican su estado mediante un mensaje Status_RESP y el $\boldsymbol{S} \boldsymbol{A E}$ los registra en el sistema.

El segundo proceso es el de solicitud de plaza dentro del cual el $N M$ solicita mediante un mensaje Space $R E Q$ enviado al $\boldsymbol{S A E}$ información para ubicar un espacio disponible para poder estacionarse (Fig. 2b). El $\boldsymbol{S A E}$ revisa la solicitud y localiza dentro de la aplicación de administración el espacio disponible más cercano a la ubicación actual del $\boldsymbol{N M}$. El $\boldsymbol{S A E}$ manda la respuesta correspondiente mediante un 
mensaje Space_RESP. El vehículo procesa la respuesta y se dirige al espacio asignado. Cuando el NMP detecta que el vehículo está estacionado envía un mensaje Parking_Status al SAE para notificarle el cambio de estado a ocupado.

El tercer proceso es el de notificación de liberación, el cual se ejecuta cuando el NMP detecta que el vehículo que estaba estacionado ha liberado el espacio (Fig. 2c). En este caso el $\boldsymbol{N M P}$ manda un mensaje Parking_Status al $\boldsymbol{S} \boldsymbol{A E}$ para notificar el cambio de estado y el $\boldsymbol{S} \boldsymbol{A} \boldsymbol{E}$ hace el registro de la actualización del nuevo estado del NMP dentro del sistema.

\subsubsection{Formato de mensajes}

Dentro del proceso de comunicación de $i-P A R K I N G$ se define una serie de formatos de tramas para el intercambio de información entre las diferentes entidades que conforman el sistema. A continuación se describen los diferentes formatos de las tramas que se envían en los diferentes procesos de comunicación (Fig. 3).

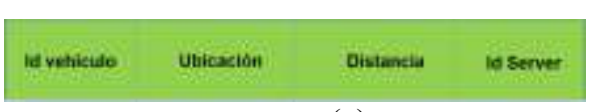

(a)

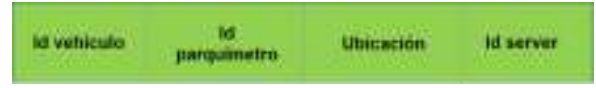

(b)

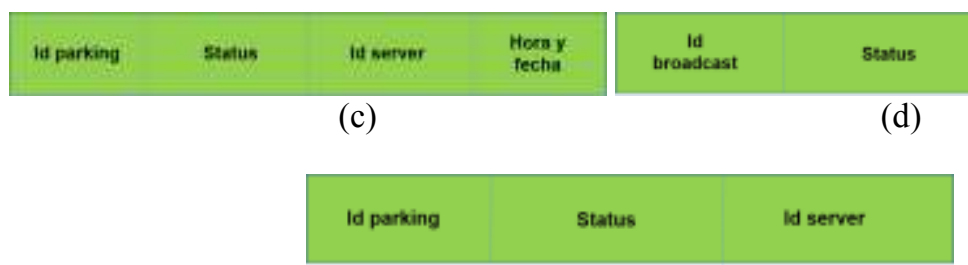

(e)

Figura 3. Representación de los formatos de trama de $i-P A R K I N G$, a) Trama Space_REQ, b) trama Space_RESP, c) Trama parking_status, d) Trama status_REQ, e) Trama space_RESP.

La trama Space_REQ es utilizada por el $\boldsymbol{N} \boldsymbol{M}$ para enviarle al $\boldsymbol{S} \boldsymbol{A E}$ una solicitud de plaza de estacionamiento (Fig. 3a). Esta trama está formada por 4 campos, los cuales explicamos a continuación. El campo Id vehículo es el identificador del vehículo que está solicitando el servicio y es asignado cuando el usuario se registra en el servicio. El campo Ubicación representa las coordenadas de GPS desde el lugar en donde se encuentra el vehículo que está solicitando el servicio. El campo Distancia representa el rango de búsqueda que establece el usuario. Este campo sirve para que el usuario pueda establecer un radio de búsqueda máximo para conseguir una plaza de estacionamiento. Finalmente Id server representa el identificador asignado al $\boldsymbol{S A E}$.

La trama Space_RESP la utiliza el $\boldsymbol{S} \boldsymbol{A E}$ para enviarle la respuesta al $\boldsymbol{N M}$ de la solicitud que realizó (Fig. 3b). Mediante esta trama el $\boldsymbol{S} \boldsymbol{A} \boldsymbol{E}$ manda la información relacionada con la plaza de estacionamiento sugerida para el $\boldsymbol{N M}$. La diferencia con la trama anterior es que ahora se le agrega el campo Id parking que representa el 
identificador asignado a cada parquímetro. Además el campo Ubicación representa las coordenadas de GPS del lugar en donde se encuentra ubicado el parquímetro.

La trama Parking_Status es utilizada en el proceso de notificación de liberación y es enviada del $\boldsymbol{N M P}$ al $\boldsymbol{S A E}$ (Fig. 3c). La trama se envía cuando el $\boldsymbol{N M P}$ detecta que la plaza que tiene asignada para su administración ha cambiado de estado. Esta trama está formada por los siguientes campos: Id parking que representa el identificador asignado a cada parquímetro. Status representa el status que recibe el servidor del parquímetro. Dicho estado puede ser 0 si la plaza está libre o 1 si la plaza está ocupada. Id server representa el identificador asignado al servidor en donde llegan las solicitudes del servicio. Hora y fecha representa el día y la hora en que el NMP detecta la liberación de la plaza.

La trama status_REQ se utiliza en el proceso de inicialización y es enviada del $\boldsymbol{S A E}$ al $\boldsymbol{N M P}$ (Fig. 3d). Esta trama está formada por los siguientes campos: Id broadcast representa que la solicitud va dirigida a todos los NMP. La dirección de Broadcast se representa con un valor de 0. Status representa el status que recibe el NMP del servidor. Cabe mencionar que este valor representa el último estado que quedó registrado en el sistema para cada parquímetro. Id server representa el identificador del SAE que realiza la solicitud.

Finalmente la trama Space_RESP es utilizada en el proceso de inicialización y es enviada del $\boldsymbol{N M P}$ al $\boldsymbol{S A E}$ (Fig. 3e). Los campos son similares, la única diferencia es que en el campus status el $\mathbf{N M P}$ notifica si la plaza que administra está ocupada o libre mediante un 0 para indicar que está libre o un 1 para indicar que está ocupada.

\section{Conclusiones}

En este trabajo presentamos la propuesta de $i-P A R K I N G$, un sistema inteligente para la gestión de plazas de estacionamiento en zonas públicas de zonas urbanas. $i$ PARKING hace uso de una red inalámbrica de sensores y comunicaciones inalámbricas para poder realizar la gestión de plazas de estacionamiento de zonas controladas por parquímetros. Este trabajo plasma el diseño completo de $i$-PARKING que se refleja en la definición de las entidades funcionales, el protocolo de comunicación y los diferentes formatos de los mensajes utilizados en el sistema.

Mediante el sistema i-Parking, se pretende asistir a los conductores en la localización de plazas de estacionamiento en zonas públicas, contribuyendo a reducir los problemas de congestionamiento e incremento de niveles de contaminación que ocasionan los conductores al conducir de forma lenta tratando de encontrar un lugar para estacionarse

\section{Referencias}

1 United Nations Population Fund.: Technical report: State of World Population 2011: People and possibilities in a world of 7 billion (2011). 
2. Department of Transportation (DOT): National Transportation Statistics, http://www.rita.dot.gov/bts/sites/rita.dot.gov.bts/files/publications/national_transportation_st atistics/index.html, (2014).

3. Lu, R., Lin, X., Zhu, H., Shen, X.: SPARK: A new VANET-based Smart Parking Scheme for Large Parking Lots. INFOCOM 2009, pp. 1413-1421, Rio de Janeiro, Brazil (2009).

4. Srikanth, V., Pramod, P., Dileep, K., Tapas, S., Mahesh, U., Patil, M, Sarat, C.: Design and implementation of a prototype Smart parking (SPARK) system using wireless sensor networks. In: International Conference on advances information networking and applications workshop, pp. 401-406, Bradford (2009).

5. Yang, G., Yang, W., Rawat, D., Olariu, S.: SmartParking: A secure and intelligent parking system. IEEE Intelligent Transportation Systems Magazine, pp. 18-30, (2011).

6. Xu, B., Wolfson, O., Yang, J. Stenneth, L., Yu, P., Nelson, P.: Real-time Street parking availability estimation. In 14th International Conference on Mobile Data Management, pp. 16-25, Milan, Italy, (2013).

7. Lu., R., Lin, X., Zhu, H., Shen, X.: An intelligent secure and privacy-preserving parking scheme through vehicular communication. IEEE Transaction on vehicular technology, pp. 2772-2785, 59(6),(2010).

8. iPARKINGS: Sistema Inteligente para control de estacionamientos. http://www.iparkings.com

9. Kokolaki, E., Karaliopoulos, M., Stavrakakis, L.: Leveraging information in parking assistance systems. IEEE Transaction on vehicular technology, pp. 4309-4317, 62(9), (2013) 